\title{
Does obesity and diabetes mellitus metastasize to the brain? "Metaboptosis" and implications for drug discovery and development
}

\author{
Roger S. McIntyre, ${ }^{1,2,3,4 *}$ (1) Carola Rong, ${ }^{1}$ (1) Rodrigo B. Mansur ${ }^{1,3}$ and Elisa Brietzke ${ }^{1}$ (1)
}

\author{
${ }^{1}$ Mood Disorders Psychopharmacology Unit (MDPU), University Health Network, University of Toronto, Toronto, ON, Canada \\ ${ }^{2}$ Brain and Cognition Discovery Foundation, Toronto, ON, Canada \\ ${ }^{3}$ Department of Psychiatry, University of Toronto, Toronto, ON, Canada \\ ${ }^{4}$ Department of Pharmacology, University of Toronto, Toronto, ON, Canada
}

Received 21 November 2018; Accepted 1 December 2018; First published 3 April 2019

The overarching aim of this commentary is to press the point that alterations in bioenergetics are hypothesized to play a critical role in the pathophysiology of many brainbased disorders (e.g., major depressive disorder, bipolar disorder). As a derivative of this hypothesis, it is further conjectured that agents capable of targeting bioenergetic effector systems may be potentially therapeutic.

Existing pharmacological treatments for mood, psychotic, as well as cognitive disorders in psychiatry, are insufficient from the point of view of achieving robust symptomatic control, functional improvement, reliable quality of life enhancement, and other patient-reported outcomes (PROs). ${ }^{1}$ Moreover, no FDA-approved agent for the foregoing disorders has demonstrated disease modification capability, the holy grail of treatment.

The cul de sac we find ourselves in with respect to psychiatric drug discovery and development, and the absence of a direct line of sight, is largely due to insufficient disease model characterization. Consequently, recapitulating disease models for mental disorders has been elusive and a priority research vista. Notwithstanding, the availability of exciting new technological approaches (e.g. inducible pluripotent stem cell research), computational statistical approaches, as well as surreal insights in neuroscience are providing the reasons to believe that future drugs in psychiatry will be not only based on disease models but possibly also modifying disease processes. ${ }^{2,3}$

Molecular, cellular, pharmacologic, animal, human, and clinical data provide convergent evidence indicating

*Address correspondence to: Roger S. McIntyre, MD, FRCPC, University Health Network; Brain and Cognition Discovery Foundation, 399 Bathurst Street, Toronto, ON M5T 2S8, Canada. (Email: Roger.McIntyre@uhn.ca) that alterations in bioenergetics are critical pathoetiologic processes within abnormal central nervous system (CNS) circuit and network function. ${ }^{4}$ For example, it is amply documented that sub-chronic and chronic alterations in central/peripheral glucose-insulin homeostasis, sirtuin, and incretin function are associated with maladaptive changes in neuronal and glial cellular architecture, morphology, number, density, and function. ${ }^{5}$

It is further observed that chronic alterations in each of the foregoing effectors result in apoptosis and epigenetic aging in animal models. ${ }^{6}$ Accelerated apoptosis is also reported in mood, psychotic, and cognitive disorders, and may in some cases be a consequence of central bioenergetic abnormalities. ${ }^{7}$ Herein, we propose the moniker "Metaboptosis" to refer to the premature cellular aging and death as a consequence of alterations in intra- and extracellular processes relevant to metabolic effector systems (glucose-insulin homeostasis). ${ }^{8}$

We also introduce the notion of obesity and type 2 diabetes mellitus (TDM2) "metastasizing" to the CNS, to both explore a unique brain-body heuristic, and more specifically, to underscore the deleterious effects that "metabolic obesity" and/or TDM2 exert on the human CNS. ${ }^{9}$ Results from both population and clinical studies in both general and mixed clinical populations indicate that metabolic obesity and TDM2 are modifiable brain hazards, as evidenced by an increased incidence of domain-based psychopathology (e.g., cognitive impairment), as well as syndromal DSM-5-defined psychopathologies (e.g., mood disorders) in affected persons. ${ }^{10-12}$

During the past decade, compelling proof-of-concept data has emerged, indicating that pharmacological treatments that target aberrant intracellular and/or 
extracellular metabolic processes exert salutary effects on neuronal cellular and sub-cellular function (e.g., mitochondria). ${ }^{13}$ The foregoing observations are replicated in animal studies with accumulating evidence indicating that appropriate "target engagement" with metabolic-based treatments exert beneficial effects, and possibly disease modifying effects, across multiple domains of psychopathology (general cognitive function).

For example, intranasal insulin, incretins (e.g., liraglutide), thiazolidinediones, caloric restriction mimetics (e.g., metformin, resveratrol), as well as SGLT-2 inhibitors, have all exhibited beneficial effects in animal models of depression, reward (e.g., motivation), and general cognitive dysfunction. ${ }^{14,15}$ It is intriguing that select agents (e.g., SGLT-2 inhibitors) have demonstrated anti-apoptotic effects in neuronal cell lines. Several of the foregoing agents have empirical evidence indicating beneficial effects in healthy control and psychiatric populations (without comorbid TDM2). ${ }^{16}$ For example, it has emerged that intranasal insulin improves measures of executive function in adults with bipolar disorder, as well as measures of memory in persons with ApoE4-mild cognitive impairment (MCI). ${ }^{17-19}$ Moreover, liraglutide demonstrates beneficial effects across several measures of cognitive function (e.g., executive function) in individuals with mood disorders, independent of its known effect on weight, and coincident with changes in CNS biomarkers of neuronal health. ${ }^{20,21}$

Drug discovery and development in psychiatry has stalled during the last 10 years, resulting in the retreat of significant research and development investment. Truly exciting developments have occurred with respect to developing glutamatergic treatments for treatmentresistant syndromes (e.g., intravenous ketamine), with the hope that related agents would be capable of providing rapid onset, robust treatment efficacy with an improved safety profile (e.g., rapastinel). ${ }^{22}$ Notwithstanding, the need exists for scalable and novel treatments for brainbased disorders that engage other (and potentially convergent) target(s) relevant to the disease process implicated in neuropsychiatric disorders.

For the practicing clinical community, we issue an alert to the brain hazards posed by metabolic obesity and TDM2 in individuals at risk or who have declared a mental disorder. The "metastasis" of peripheral metabolic alterations to the brain introduces a set of both preventive and pre-emptive opportunities that are costeffective, and likely to be clinically relevant and acceptable to most patients (e.g., sleep hygiene, healthier diet, exercise). For both the academic/research and investment communities, ample evidence indicates that developing and/or repurposing pharmacologic agents that engage metabolic systems may not only be symptom suppressing but disease modifying for mood, psychotic, and cognitive disorders.

\section{Disclosures}

Dr. McIntyre reports grants from Lundbeck, Shire, Purdue, and Allergan; speaking fees from Shire, Purdue, Otsuka, Janssen-Ortho, Lundbeck, Pfizer, Neurocrine, Neuralstem, Sunovion, Takeda, and Allergan outside the submitted work.

Dr. Mansur reports grants from Department of Psychiatry, University of Toronto and Canadian Institutes of Health Research; honorarium from Medical Psychotherapy Association Canada; logistical support from Medical Psychotherapy Association Canada, outside the submitted work.

Dr. Brietzke reports research funding from FAPESP, CNPq, and CAPES; financial support from L'Oreal for Women in Science Award; speaker fees from Daiichi-Sankyo; honoraria from Sanofi, Europharma, Ache, Astra-Zeneca, and Daiichi-Sankyo, outside the submitted work.

Dr. Rong has nothing to disclose.

\section{REFERENCES:}

1. McIntyre RS. A vision for drug discovery and development: Novel targets and multilateral partnerships. Adv Ther. 2014; 31(3): 245-246.

2. Wen Z, Christian KM, Song H, et al. Modeling psychiatric disorders with patient-derived iPSCs. Curr Opin Neurobiol. 2016; 36: 118-127.

3. O'shea KS, McInnis MG. Neurodevelopmental origins of bipolar disorder: iPSC models. Mol Cell Neurosci. 2016; 73: 63-83.

4. Villa RF, Ferrari F, Bagini L, et al. Mitochondrial energy metabolism of rat hippocampus after treatment with the antidepressants desipramine and fluoxetine. Neuropharmacology. 2017; 121: 30-38.

5. Srivastava R, Faust T, Ramos A, et al. Dynamic changes of the mitochondria in psychiatric illnesses: New mechanistic insights from human neuronal models. Biol Psychiatry. 2018; 83: 751-760. doi: 10.1016/j.biopsych.2018.01.007.

6. Pietruczuk K, Lisowska KA, Grabowski K, et al. Proliferation and apoptosis of $\mathrm{T}$ lymphocytes in patients with bipolar disorder. Sci Rep. $2018 ; \mathbf{8}(1)$ : 3327.

7. Neth BJ, Craft S. Insulin resistance and Alzheimer's disease: Bioenergetic linkages. Front Aging Neurosci. 2017; 9: 345.

8. Zheng K, Wang N, Shen Y, et al. Pro-apoptotic effects of microRNA-365 on retinal neurons by targeting IGF-1 in diabetic rats: An in vivo and in vitro study. J Diabetes Investig. 2018; 9(5): 1041-1051. doi: 10.1111/jdi.12815.

9. Kalaivani A, Uddandrao VS, Parim B, et al. Reversal of high fat diet-induced obesity through modulating lipid metabolic enzymes and inflammatory markers expressions in rats. Arch Physiol Biochem. 2018; 124: 1-7.

10. Mansur RB, Brietzke E, McIntyre RS. Is there a 'metabolic-mood syndrome'? A review of the relationship between obesity and mood disorders. Neurosci Biobehav Rev. 2015; 52: 89-104.

11. Mansur RB, Lee Y, Zhou AJ, et al. Determinants of cognitive function in individuals with type 2 diabetes mellitus: A meta-analysis. Ann Clin Psychiatry. 2018; 30(1): 38-50.

12. McIntyre RS, Soczynska JK, Konarski JZ, et al. Should depressive syndromes be reclassified as 'metabolic syndrome type II'? Ann Clin Psychiatry. 2007; 19(4): 257-264.

13. Skvarc DR, Dean OM, Byrne LK, et al. The effect of N-acetylcysteine (NAC) on human cognition - A systematic review. Neurosci Biobehav Rev. 2017; 78: 44-56. 
14. Sa-Nguanmoo P, Tanajak P, Kerdphoo S, et al. SGLT2-inhibitor and DPP-4 inhibitor improve brain function via attenuating mitochondrial dysfunction, insulin resistance, inflammation, and apoptosis in HFD-induced obese rats. Toxicol Appl Pharmacol. 2017; 333: 43-50.

15. Colle R, de Larminat D, Rotenberg S, et al. Pioglitazone could induce remission in major depression: A meta-analysis. Neuropsychiatr Dis Treat. 2017; 13: 9-16

16. Colle R, de Larminat D, Rotenberg S, et al. PPAR- $\gamma$ agonists for the treatment of major depression: A review. Pharmacopsychiatry. 2017; 50(2): 49-55.

17. McIntyre RS, Soczynska JK, Woldeyohannes HO, et al. A randomized, double-blind, controlled trial evaluating the effect of intranasal insulin on neurocognitive function in euthymic patients with bipolar disorder. Bipolar Disord. 2012; 14(7): 697-706.

18. Craft S, Claxton A, Baker LD, et al. Effects of regular and long-acting insulin on cognition and Alzheimer's disease biomarkers: A pilot clinical trial. J Alzheimer Dis. 2017; 57(4): 1325-1334.
19. Claxton A, Baker L, Hanson A, et al. Long-acting intranasal insulin detemir improves working memory for adults with mild cognitive impairment or early-stage Alzheimer's dementia. Alzheimers Dementia: J Alzheimers Assoc. 2013; 9(4): P657.

20. Mansur RB, Ahmed J, Cha DS, et al. Liraglutide promotes improvements in objective measures of cognitive dysfunction in individuals with mood disorders: A pilot, open-label study. J Affect Disord. 2017; 207: 114-120.

21. Mansur RB, Lee Y, Subramaniapillai M, et al. Cognitive dysfunction and metabolic comorbidities in mood disorders: A repurposing opportunity for glucagon-like peptide 1 receptor agonists? Neuropharmacology. 2018; 136: 335-342. doi: 10.1016/j. neuropharm.2018.01.048.

22. Phillips AG, Geyer MA, Robbins TW. Effective use of animal models for therapeutic development in psychiatric and substance use disorders. Biol Psychiatry. 2018; 83: 915-923. doi: 10.1016/j. biopsych.2018.01.014 